\title{
Force-displacement relation in a tangential frictional contact with adhesion
}

Cite as: AIP Conference Proceedings 2051, 020244 (2018); https://doi.org/10.1063/1.5083487

Published Online: 12 December 2018

Valentin L. Popov

\section{ARTICLES YOU MAY BE INTERESTED IN}

Theory of adhesion: Role of surface roughness

The Journal of Chemical Physics 141, 124701 (2014); https://doi.org/10.1063/1.4895789

Theory of rubber friction and contact mechanics

The Journal of Chemical Physics 115, 3840 (2001); https://doi.org/10.1063/1.1388626

Features of recrystallization during annealing of nanocrystalline Ti-50.9 at. \% Ni alloy AIP Conference Proceedings 2051, 020243 (2018); https://doi.org/10.1063/1.5083486
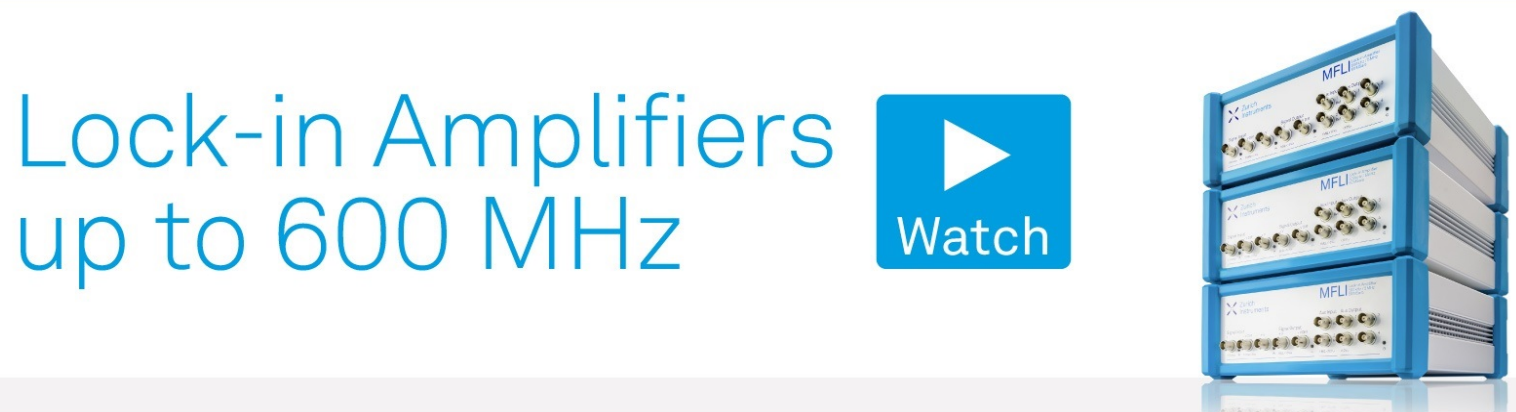


\title{
Force-Displacement Relation in a Tangential Frictional Contact with Adhesion
}

\author{
Valentin L. Popov \\ Berlin University of Technology, Berlin, 10623 Germany \\ v.popov@tu-berlin.de
}

\begin{abstract}
We consider tangential contact between a rigid cylinder and elastic half-space in the presence of adhesion and Coulomb's frictional force. In the limit of very small range of adhesive interaction, the main governing dimensionless parameters are identified and it is shown that the shape of the relation between the normalized force and normalized displacement is function of only one system parameter closely related to the Tabor parameter. However, the qualitative behavior is the same for arbitrary values of the Tabor parameter: the force monotonously increases from zero to the maximum value corresponding to the complete sliding. This behavior is qualitatively different from that known in the case of non-adhesive contact where - in the case of flat-ended cylindrical punch - the whole contact area remains in stick state until the displacement achieves some critical value, after which complete sliding starts.
\end{abstract}

\section{INTRODUCTION}

As noted in [1], in JKR theory [2], the total energy of an adhesive contact does not depend on the tangential displacement; thus the JKR contact does not formally possess any "tangential strength". Yet the lack of tangential strength of adhesive contacts is obviously contradicted by experimental results. The physical cause of this contradiction lies in the heterogeneous structure found at the microscopic scale (or at the atomic scale, at the latest) of any real interface. This heterogeneity leads to appearance of frictional force and to a finite contact strength in the tangential direction. The problem of adhesive contact with friction was discussed recently in [3]. In [1, 4], the frictional tangential contact with adhesion was considered under the simplifying assumption that the adhesive forces create additional macroscopic pressure in the contact, which, according to Coulomb's law of friction, leads to increased friction forces. In the present paper we first recapitulate briefly the main findings of the above works and then analyze in more detail the dependency of the tangential force on tangential displacement in the simplest case of a contact between an elastic half-space and a flat-ended cylindrical indenter.

\section{ADHESIVE TANGENTIAL CONTACT}

In this paper, we will restrict ourselves to the simplest model of an adhesive tangential contact problem with a properly defined "adhesion" in the normal direction and "friction" in the horizontal direction. We assume that the adhesive forces have sufficient range to be considered "macroscopic" with regard to the friction forces in the contact. However, this range is considered to be smaller than any other length of the contact problem. Since both the normal and the tangential contact problem of two elastic bodies can be reduced to the contact between a rigid body and an elastic half-space (with modified material properties), we will consider-without loss of generality - the case of a rigid indenter in contact with an elastic half-space. We consider the frictionless contact between two elastic bodies with elasticity moduli $E_{1}$ and $E_{2}$ and Poisson's ratios $v_{1}$ and $v_{2}$ as well as shear moduli $G_{1}$ and $G_{2}$. The difference between the profiles is $\tilde{z}=f(r)$, where $r$ is the polar radius of the contact plane. This contact problem is 
equivalent to the contact of a rigid indenter with the profile $\tilde{z}=f(r)$ and an elastic half-space with the effective elasticity moduli $E^{*}$ and [5]:

$$
\frac{1}{E^{*}}=\frac{1-v_{1}}{2 G_{1}}+\frac{1-v_{2}}{2 G_{2}}, \frac{1}{G^{*}}=\frac{2-v_{1}}{4 G_{1}}+\frac{2-v_{2}}{4 G_{2}} .
$$

For the adhesive forces, we use the model of Dugdale, where the adhesive pressure remains constant and equal to $\sigma_{0}$ up to a certain distance $h$ between the surfaces and abruptly drops to zero after that distance. The theory of adhesive contacts for this particular interaction was created by Maugis [6] but we will use the formulation provided in [1]. This formulation is based on the Method of Dimensionality Reduction [7] and uses transformation in the MDR-space. According to the MDR, the complete information about the contact is "condensed" in the equivalent profile $g(x)$ :

$$
g(x)=|x|_{0}^{|x|} \frac{f^{\prime}(r)}{\sqrt{x^{2}-r^{2}}} \mathrm{~d} r .
$$

In [1], it was shown that in the limit of very small range of interaction of adhesive forces, the following relations are valid for the relationship between tangential displacement $u^{(0)}$ of the indenter and the radius of the stick zone $c$ :

$$
\frac{G^{*} u^{(0)}}{\mu}=\sqrt{4 \sigma_{0}^{2}\left(a^{2}-c^{2}\right)+2 \pi a E^{*} \Delta \gamma}-\sqrt{2 \pi a E^{*} \Delta \gamma}+E^{*}[g(a)-g(c)]
$$

where $\mu$ is the coefficient of friction, $a$ is the contact radius, and $\Delta \gamma=\sigma_{0} h$ is the specific work of separation (work of adhesion per unit area).

The same approximation yields for the tangential force

$$
\begin{gathered}
\frac{F_{x}}{\mu}=c \sqrt{4 \sigma_{0}^{2}\left(a^{2}-c^{2}\right)+2 \pi a E^{*} \Delta \gamma}-a \sqrt{2 \pi a E^{*} \Delta \gamma}+2 E^{*}[a g(a)-c g(c)] \\
+\pi \sigma_{0} a^{2}\left(1-\frac{2}{\pi} \arcsin \frac{c}{a}\right)-2 E^{*} \int_{c}^{a} g(x) \mathrm{d} x .
\end{gathered}
$$

\section{TANGENTIAL CONTACT OF A FLAT-ENDED CYLINDER}

For a flat-ended cylinder with radius $a$, the equivalent profile given by (2) is especially simple:

$$
g(x)=\left\{\begin{array}{l}
0,|x| \leq a \\
\infty,|x|>a .
\end{array}\right.
$$

For this case, Eqs. (3) and (4) can be rewritten as

$$
\begin{gathered}
\frac{G^{*} u^{(0)}}{\mu}=\sqrt{4 \sigma_{0}^{2}\left(a^{2}-c^{2}\right)+2 \pi a E^{*} \Delta \gamma}-\sqrt{2 \pi a E^{*} \Delta \gamma}, \\
\frac{F_{x}}{\mu}=c \sqrt{4 \sigma_{0}^{2}\left(a^{2}-c^{2}\right)+2 \pi a E^{*} \Delta \gamma}-a \sqrt{2 \pi a E^{*} \Delta \gamma}+\pi \sigma_{0} a^{2}\left(1-\frac{2}{\pi} \arcsin \frac{c}{a}\right) .
\end{gathered}
$$

Introducing dimensionless variables

$$
\tilde{u}^{(0)}=\frac{u^{(0)}}{\mu \sqrt{2 \pi a h E^{*} \sigma_{0} / G^{* 2}}}, \quad \tilde{F}_{x}=\frac{F_{x}}{\mu a \sqrt{2 \pi a E^{*} \sigma_{0} h}}, \xi=\frac{\sigma_{0} a}{E^{*} h}, \tilde{c}=\frac{c}{a},
$$

we can rewrite Eqs. (6) and (7) in the form 


$$
\begin{gathered}
\tilde{u}^{(0)}=\sqrt{\frac{2}{\pi} \xi\left(1-\tilde{c}^{2}\right)+1}-1, \\
\tilde{F}_{x}=\tilde{c} \sqrt{\frac{2}{\pi} \xi\left(1-\tilde{c}^{2}\right)+1}-1+\sqrt{\frac{\pi}{2} \xi}\left(1-\frac{2}{\pi} \arcsin \tilde{c}\right) .
\end{gathered}
$$

These two equations determine in implicit form the interrelation between dimensionless displacement and force. One can easily see that the form of this dependence is determined by only one dimensionless parameter $\xi$. Note that the dimensionless parameter $\xi$ has simple physical meaning: it relates the length of the critical "adhesive neck" (order of magnitude $\sigma_{0} a / E^{*}$ ) and the acting rage of adhesive forces, $h$, and thus is closely related to the so-called Tabor-parameter [8]. Dependency of the normalized force on the normalized displacement is shown in Fig. 1.

The maximum value of tangential displacement $\tilde{u}^{(0)}$ at the moment when the complete sliding starts and the corresponding value of the tangential force can be found from Eqs. (9) and (10) by setting $c=0$ :

$$
\begin{gathered}
\tilde{u}^{(0)}=\sqrt{\frac{2}{\pi} \xi+1}-1, \\
\tilde{F}_{x}=\sqrt{\frac{\pi}{2} \xi}-1
\end{gathered}
$$

or in dimensional form

$$
\begin{gathered}
u^{(0)}=\mu \sqrt{2 \pi a h \frac{E^{*} \sigma_{0}}{G^{* 2}}}\left(\sqrt{\frac{2}{\pi} \frac{\sigma_{0} a}{E^{*} h}+1}-1\right), \\
F_{x}=\mu a \sqrt{2 \pi a E^{*} \sigma_{0} h}\left(\sqrt{\frac{\pi}{2} \frac{\sigma_{0} a}{E^{*} h}}-1\right) .
\end{gathered}
$$

One can see that the shape of curves shown in Fig. 1 which correspond to various $\xi$-values is very similar. We therefore plotted the dependencies once more in Fig. 2 showing the tangential force normalized by its maximum value (14) against displacement normalized by its maximum value (13). In these variables, all the curves "condense" to a more or less unique "master curve".

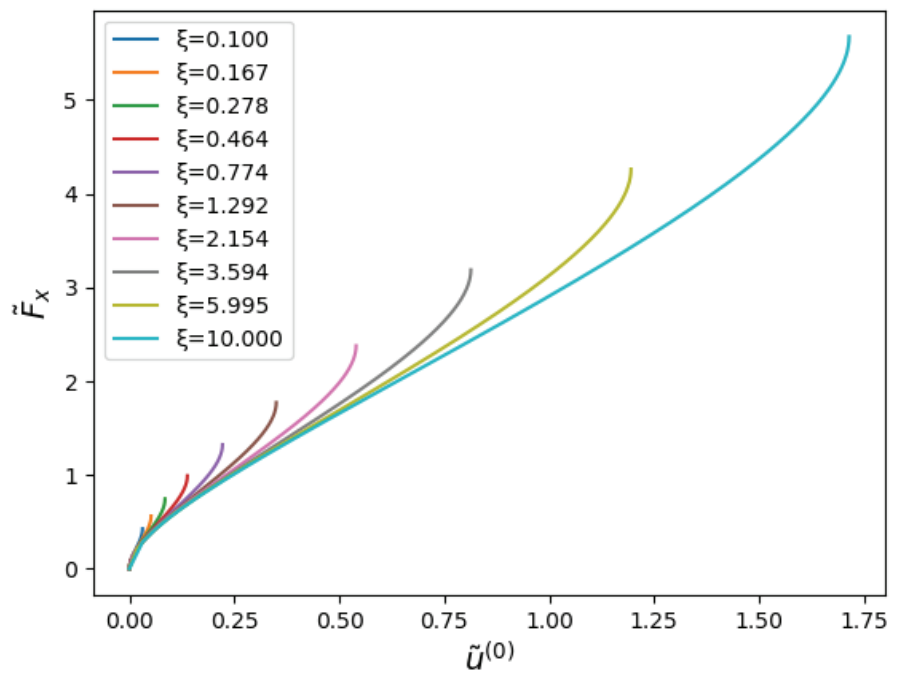

FIGURE 1. Dependency of the normalized force on the normalized displacement for values of parameter $\xi$ ranging from 0.1 to 10 


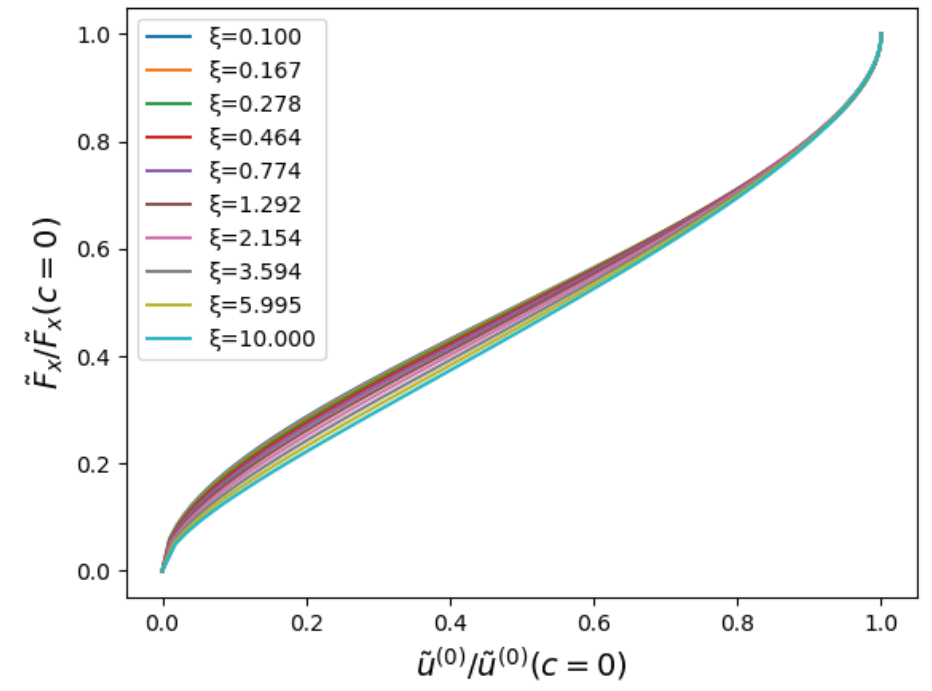

FIGURE 2. Dependencies on the tangential force on tangential displacement (both normalized to the corresponding maximum values)

\section{CONCLUSION}

We considered tangential contact between a flat-ended cylinder and an elastic half-space in the simultaneous presence of adhesive and frictional forces. The relation between the normalized tangential force and the normalized tangential displacement is dependent only on one parameter $\xi=\sigma_{0} a /\left(E^{*} h\right)$, which is very closely related to the Tabor parameter [8]. The qualitative behavior is the same for arbitrary values of the Tabor parameter: the force monotonously increases from zero to the maximum value corresponding to the complete sliding. This behavior is different from that known in the case of non-adhesive contact (Cattaneo [9] and Mindlin [10]) where-in the case of flat-ended cylindrical punch - the whole contact area remains in stick state until the displacement achieves some critical value, after which complete sliding starts.

\section{ACKNOWLEDGMENTS}

The author acknowledges financial support from the Deutsche Forschungsgemeinschaft (DFG, project PO 81055-1). The author thanks M. Popov for helpful discussions and help in editing the paper.

\section{REFERENCES}

1. V. Popov, M. Heß, and E. Willert, Handbook of Contact Mechanics, Springer, 2018.

2. K. L. Johnson, K. Kendall, and A. D. Roberts, Proc. Royal Soc. London A 324, 301-313 (1971).

3. N. Menga and G. Carbone, "Adhesive elastic periodic contacts: the role of interfacial friction and slab thickness", in EUROMECH COLLOQUIUM Contact Mechanics and Coupled Problems in Surface Phenomena (2015).

4. V. L. Popov and A. V. Dimaki, J. Adhes. 93, 1131-1145 (2016).

5. H. Hertz, J. Rreine Angew Math. 1882, 156-171 (1882).

6. D. Maugis, J. Colloid Interf. Sci. 150, 243-269 (1992).

7. V.L. Popov and M. Hess, Method of Dimensionality Reduction in Contact Mechanics and Friction (Springer, 2015).

8. D. Tabor, J. Colloid Interf. Sci. 58, 2-13 (1977).

9. C. Cattaneo, Rendiconti dell' Academi Nazionale dei Lincei, 27, 342-348, 434-436, 474-478 (1938).

10. R. D. Mindlin, J. Appl. Mech. 16, 259-268 (1949). 